\title{
Inhibition of NF-kB and Akt pathways by an antibody-avidin fusion protein sensitizes malignant B-cells to cisplatin-induced apoptosis
}

\author{
ERIKO SUZUKI ${ }^{1 *}$, TRACY R. DANIELS ${ }^{3 *}$, GUSTAVO HELGUERA ${ }^{3}$, MANUEL L. PENICHET $^{3,4}$, \\ KAZUO UMEZAWA ${ }^{2}$ and BENJAMIN BONAVIDA ${ }^{4}$ \\ ${ }^{1}$ Department of Urology, School of Medicine, Keio University, 35 Shinanomachi, Shinjuku-ku, Tokyo 165-8582; \\ ${ }^{2}$ Department of Applied Chemistry, Keio University, 3-14-1 Hiyoshi, Kohoku-ku, Yokohama 223-0061, Japan; \\ Departments of ${ }^{3}$ Surgery, Division of Surgical Oncology, ${ }^{4}$ Microbiology, Immunology and Molecular Genetics, \\ David Geffen School of Medicine, The Molecular Biology Institute, Jonsson Comprehensive Cancer Center, \\ University of California at Los Angeles, 10833 Le Conte Avenue, Los Angeles, CA 90095, USA
}

Received January 4, 2010; Accepted February 1, 2010

DOI: 10.3892/ijo_00000615

\begin{abstract}
Multiple myeloma (MM) is an incurable disease of malignant plasma cells. Recent therapeutic advancements have resulted in improved response rates, however, there is no improvement in overall survival, therefore, new therapeutics are needed. Since the transferrin receptor is upregulated on the surface of MM cells, we previously developed an antibody fusion protein consisting of an IgG3 specific for the human transferrin receptor 1 (TfR1, CD71) genetically fused to avidin at its carboxy-terminus (ch128.1Av). We have previously shown that ch128.1Av exhibits intrinsic cytotoxicity against certain malignant B-cells by disrupting the cycling of the TfR and decreasing TfR cell surface expression resulting in lethal iron starvation. In addition, ch128.1Av can sensitize malignant cells to apoptosis induced by gambogic acid, a herbal drug used in Chinese medicine. In this study, we hypothesized that ch128.1Av may also sensitize drug-resistant malignant B-cells to chemotherapeutic agents by inhibiting key survival pathways. In this study we show that ch128.1 Av sensitizes malignant B-cells to apoptosis induced by cisplatin (CDDP). The sensitization by ch128.1Av resulted in the inhibition of the constitutively activated Akt and NF- $\mathrm{KB}$ survival/antiapoptotic pathways and downstream decreased expression of antiapoptotic gene products such as $\mathrm{Bcl}_{\mathrm{xL}}$ and survivin. The direct role of the inhibition of the Akt and NF- $\mathrm{B}$ pathways by ch128.1Av in CDDP-mediated cytotoxicity was demonstrated
\end{abstract}

Correspondence to: Professor Benjamin Bonavida, Department of Microbiology, Immunology and Molecular Genetics, 10833 Le Conte Avenue, Los Angeles, CA 90095, USA

E-mail: bbonavida@mednet.ucla.edu

*Contributed equally

Key words: antibody fusion protein, transferrin receptor, NF-kB, Akt by the use of specific chemical inhibitors and siRNA which mimicked the effects of ch128.1Av. Overall, this study provides evidence of the therapeutic potential of ch128.1Av as a chemo-sensitizing agent in drug-resistant tumor cells.

\section{Introduction}

Increasing knowledge of the biology of multiple myeloma (MM) is already contributing to the design of new drugs that target cellular proteins or pathways involved in the pathophysiology of this disease. These agents include inhibitors of tyrosine-kinase receptors (TKR) (1), agents acting through cell-surface receptors such as monoclonal antibodies against plasma-cell antigens (2), and inhibitors of signaling pathways such as NF- $\mathrm{kB}$ inhibitors (3-5) and Akt inhibitors $(6,7)$. Patients with $\mathrm{MM}$ are treated with chemotherapy and recently with the proteasome inhibitor Bortezomib (8). However, there are patients who either do not respond to such treatments or develop resistance. Therefore, novel therapeutic approaches are needed as alternative strategies to treat this disease.

Transferrin primarily functions as an iron transporter through the blood. The transferrin receptor 1 (TfR1, CD71) is ubiquitously expressed on normal cells at various levels (9). TfR 1 expression is increased in cells with a high proliferation rate or on cells that require large amounts of iron. In normal tissues, the constitutive expression of the TfR 1 is limited to the liver, epidermis, intestinal epithelium, vascular endothelium of brain capillaries, and certain populations of blood cells in the bone marrow (9). In contrast, high levels of TfR1 expression have been identified in many tumors including MM (9). Due to its pivotal role in iron uptake, TfR 1 is expressed more abundantly in malignant cells compared to normal cells and in many cases, this increased expression correlates with tumor stage and poor prognosis $(9,10)$. Due to its extracellular accessibility and its upregulation on the surface of malignant cells, the TfR1 has been widely used for the delivery of many therapeutics into cancer cells by receptormediated endocytosis (11). 
In order to target the TfR1, we previously generated a mouse/human chimeric antibody fusion protein specific for TfR1 (12-14). This fusion protein (ch128.1Av) contains the variable regions of the murine antibody 128.1 and human IgG3 constant regions that are genetically fused to avidin at the carboxy-terminus of the heavy chains. This molecule was originally designed as a delivery vehicle with the ability to deliver many biotinylated anti-tumor compounds into cancer cells. We have previously shown that ch128.1 delivers active biotinylated molecules into cancer cells, including saporin, a toxin derived from the plant Saponaria officinalis $(12,15)$. We have also shown that ch128.1Av exhibits intrinsic cytotoxic activity alone against certain malignant B-cells $(13,16)$. This cytotoxicity is due to a disruption of the constitutive TfR1 cycling pathway that leads to decreased TfR1 surface expression and ultimately, after at least $48 \mathrm{~h}$, lethal through iron deprivation (13). More recently, we have demonstrated that ch128.1Av enhances the cytotoxicity of gambogic acid, a traditional Chinese medicine that can also bind the TfR1 (16). The antibody fusion protein can also sensitize malignant B-cells to gambogic acid-induced apoptosis (16).

In the current study, we hypothesized that ch128.1Av may also sensitize malignant B-cells to more traditional chemotherapeutic drugs. This hypothesis was tested and the following were investigated: a) Does the combination treatment of ch128.1Av and the chemotherapeutic drug cisdiamminedichloroplatinum (II) (CDDP, also known as cisplatin) result in enhanced cytotoxic effects? b) Does ch128.1Av-mediated sensitization result from inhibition of constitutively activated cell survival/anti-apoptotic pathways such as the NF- $\mathrm{kB}$ and Akt pathways? c) Does inhibition of NF- $\mathrm{BB}$ activity by ch128.1Av mediate, in part, CDDP-induced sensitization to apoptosis? Does the NF- $\mathrm{B}$ inhibitor, DHMEQ, mimic ch128.1Av sensitization to CDDP? d) Is ch128.1 Avinduced inhibition of Akt activity responsible, in part, for the sensitization of cancer cells to CDDP-induced apoptosis? Does the Akt chemical inhibitor LY294002 or Akt siRNA mimic ch128.1Av sensitization to CDDP? and e) Does the treatment of malignant B-cells with ch128.1Av involve mitochondrial signaling for apoptosis? The findings presented herein support the above hypothesis.

\section{Materials and methods}

Reagents. RPMI-1640, opti-MEM and fetal bovine serum (FBS) were purchased from Invitrogen (Carlsbard, CA, USA). LY294002 was purchased from Merck Japan (Tokyo, Japan). DHMEQ was a kind gift from Dr K. Umezawa (Keio University, Japan). Anti-Bcl $\mathrm{xL}_{\mathrm{L}}$, anti-cIAP1, anti-PARP, anti-caspase 9, anti-survivin, anti-human phospho-Akt (Ser473), anti-Akt and anti- $\beta$-actin antibodies were obtained from Cell Signaling Technology (Beverly, MA, USA). Secondary HRP-conjugated anti-rabbit or anti-mouse IgG antibodies, Akt siRNA and control scramble siRNA were purchased from Sigma-Aldrich (St. Louis, MO, USA). Protein A-agarose was purchased from Pierce (Rockford, IL, USA). The Annexin V-FITC kit was purchased from Beckman Coulter (Marseille, France).

Antibody-avidin fusion protein. ch128.1Av has been described previously (12). Briefly, this molecule was expressed in the murine myeloma cell line $\mathrm{Sp} 2 / 0-\mathrm{Ag} 14$ and purified from culture supernatants by using affinity chromatography as described $(12,17)$. Purity was assessed by Simply blue (Invitrogen) staining of SDS-PAGE gels. All protein concentrations were determined by the bicinchoninic acid based protein assay (BCA Protein Assay, Thermo Fisher Scientific, Rockford, IL, USA) and ELISA as described (17).

Cell lines. The human cell lines IM-9 (EBV-transformed B-lymphoblastoid cell line originally isolated from the blood of a patient with multiple myeloma) and RPMI-8226 (a multiple myeloma cell line also isolated from the blood of a patient with multiple myeloma) were obtained from Human Science Technology (Osaka, Japan). Cells were cultured at $37^{\circ} \mathrm{C}, 5 \% \mathrm{CO}_{2}$ in RPMI-1640 medium supplemented with $10 \% \mathrm{FBS}, 1 \% \mathrm{NaHCO}_{3}$ and $1 \%$ penicillin, streptomycin (v/v).

Determination of apoptosis by flow cytometry. Apoptosis was detected by Annexin V/Propidium Iodide staining followed by flow cytometry analysis as described previously (18).

Measurement of mitochondrial membrane depolarization. The mitochondria-specific dye 3,3-dihexyloxacarbocyanine $\left(\right.$ DiOC $_{6}$; Invitrogen) was used to measure the mitochondrial membrane potential in both cell lines before and after $24 \mathrm{~h}$ of treatment with ch128.1Av as previously reported (19).

Western blotting. IM-9 and 8226 cells $\left(4 \times 10^{5}\right.$ cells $\left./ \mathrm{ml}\right)$ were seeded in a $100-\mathrm{mm}$ dish containing $9 \mathrm{ml}$ of the complete medium. The cells were treated with indicated concentrations of ch128.1Av for $24 \mathrm{~h}$, washed 2 times with PBS, and then treated with $10 \mu \mathrm{g} / \mathrm{ml}$ of CDDP for an additional $24 \mathrm{~h}$. The cells were harvested in PBS on ice and centrifuged at 3,500 rpm for $5 \mathrm{~min}$. The cells were lysed in $100 \mu \mathrm{l}$ of lysis buffer (1\% Triton X-100, $20 \mathrm{mM}$ Tris, $137 \mathrm{mM} \mathrm{NaCl,} 1 \mathrm{mM}$ PMSF, $2 \mathrm{mM} \mathrm{Na}_{3} \mathrm{VO}_{4}$, and $10 \mu \mathrm{g} / \mathrm{ml}$ leupeptin). The cell lysate was centrifuged at $15,000 \mathrm{x} g$ for $10 \mathrm{~min}$, and the supernatant was used for further analysis. Protein concentrations were determined using the Coomassie Brilliant Blue Method. The proteins were denatured at $95^{\circ} \mathrm{C}$ for $5 \mathrm{~min}$ in loading buffer (60 mM Tris, 2.5\% SDS, 10\% glycerol, 5\% 2-ME, and $0.01 \%$ bromophenol blue). Aliquots containing $70 \mu \mathrm{g}$ of total protein from each sample were separated by SDS-PAGE and transferred onto a PVDF membrane at $200 \mathrm{~mA}$ for $1 \mathrm{~h}$. After the transfer, the membrane was washed for $30 \mathrm{~min}$ at room temperature in a TBS-Tween buffer $(20 \mathrm{mM}$ Tris- $\mathrm{HCl}$, pH 7.6, containing $137 \mathrm{mM} \mathrm{NaCl}$ and $0.1 \%$ Tween-20) supplemented with $5 \%$ non-fat milk. The membrane was washed three times with TBS-Tween and probed for $1 \mathrm{~h}$ with the primary antibody diluted at $1 / 300$ with $5 \%$ non-fat milk in TBS-Tween. After the membrane had been washed 6 times with TBS-Tween, the membrane was incubated with a secondary HRP-conjugated anti-rabbit or anti-mouse IgG antibodies diluted at 1/3000 with 5\% non-fat milk TBS-Tween for $1 \mathrm{~h}$ at room temperature. Finally, after the membrane had been washed with TBS-Tween eight times the proteins were visualized on X-ray film with an ECL Western blotting detection kit (Perkin Elmer Life Sciences, Tokyo, Japan).

Transfection with siRNA. Cells were cultured in $3 \mathrm{ml}$ of OptiMEM medium supplemented with FBS. Transfections with 
targeted siRNA (Sigma-Aldrich) or control scramble siRNA (Sigma-Aldrich) were performed using the Xtreme GENE reagent (Roche Diagnostics Corporation, Indianapolis, IN, USA), according to the manufacturer's instructions. To determine Akt siRNA-induced sensitization to CDDP-induced apoptosis following transfection, the cells were treated with CDDP for an additional $24 \mathrm{~h}$, then were processed for the Annexin V/Propidium Iodide staining and analyzed by flow cytometry as described above.

Analysis of DNA binding activity. DNA binding activity was measured by using the Trans AM ${ }^{\mathrm{TM}}$ Transcription Factor Assays kit (Active Motif, Tokyo, Japan) according to the manufacturer's instructions.

Statistical analysis. Assays were set up in triplicates and the results are expressed as the mean \pm SD. Statistical analysis was performed using the Student's t-test.

\section{Results}

ch128.1Av-induced sensitization of malignant B-cell lines to apoptosis by CDDP. The malignant B-cell lines IM-9 and 8226 were used as model systems to investigate the effect of ch128.1Av on the response of cancer cells to chemotherapeutic drugs such as CDDP. The IM-9 and 8226 cell lines were treated with different concentrations of ch128.1Av for $24 \mathrm{~h}$ and followed by treatment with CDDP $(10 \mu \mathrm{g} / \mathrm{ml})$ for an additional $24 \mathrm{~h}$. The cells were then examined for apoptosis. While treatment with ch128.1Av alone or CDDP alone resulted in no significant apoptosis, the combination treatment resulted in significant potentiation of apoptosis (Fig. 1). By comparison, the sensitization of the cell line 8226 was more potent than the sensitization of the IM-9 cell line. These findings demonstrate that ch128.1Av is able to modify the apoptotic pathway(s) in the cell lines by reducing the threshold of resistance so that CDDP treatment can trigger apoptosis. We then examined the potential underlying mechanism by which ch128.1Av signals the cells and results in chemosensitization.

Inhibition of the constitutively activated $N F-\kappa B$ pathway in malignant B-cells by ch128.1Av. The above findings of ch128.1Av-mediated sensitization to CDDP apoptosis may have resulted from the inhibition of anti-apoptotic gene products that are regulated by the $\mathrm{NF}-\kappa \mathrm{B}$ pathway. To test this hypothesis, the cancer cells were treated with various concentrations of ch128.1Av for $24 \mathrm{~h}$ and cell lysates were prepared for Western blot analysis. Treatment of IM-9 cells with ch128.1 Av resulted in a significant inhibition of the activation of NF- $\mathrm{KB}$ as evidenced by the decrease in phosphop65 at concentrations $\geq 16 \mathrm{nM}$ ch128.1Av and complete inhibition of phosphorylation at $32 \mathrm{nM}$ (Fig. 2A, left panel). There was no effect on total p65 protein level, suggesting that the effect of ch128.1Av occurs at the activation level and not at the expression level. B-actin was used as a loading control. For 8226 cells, there was significant decrease in the levels of phospho-p65 at $\geq 8 \mathrm{nM}$ ch128.1Av and more inhibition at $32 \mathrm{nM}$ (Fig. 2A, right panel). Like IM-9, there was no effect on total p65 protein levels.

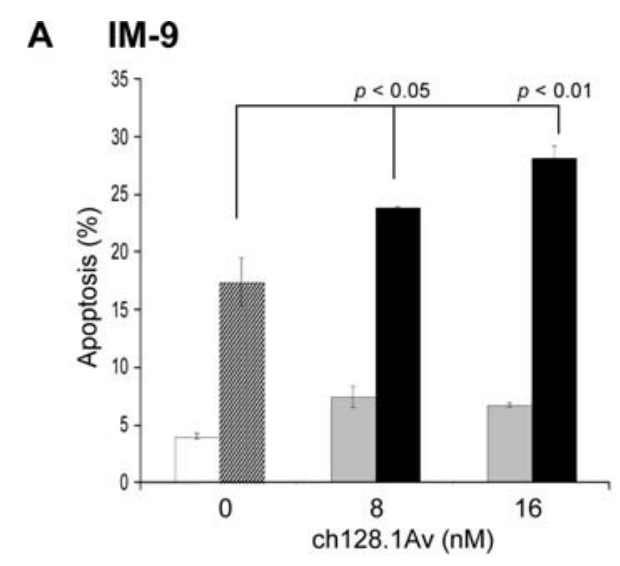

\section{B $\quad 8226$}

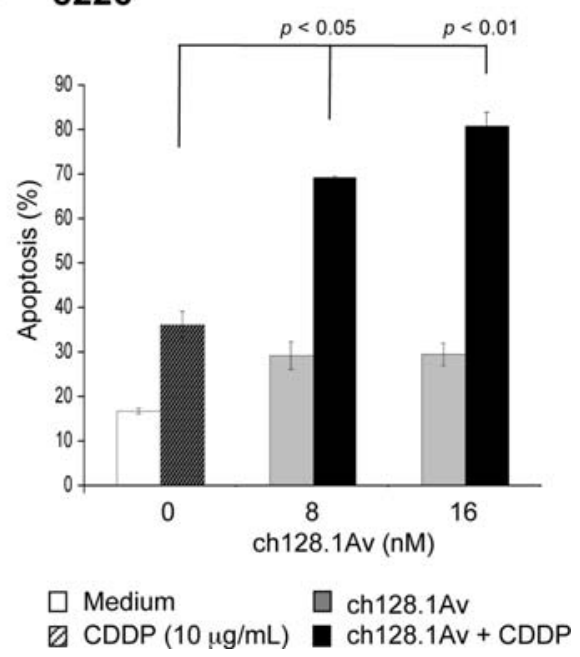

Figure 1. ch128.1Av sensitizes tumor cells to CDDP-induced apotosis. IM-9 (A) and 8226 cells (B) were pre-treated with various concentrations of ch128.1Av for $24 \mathrm{~h}$ followed by treatment with CDDP $(10 \mu \mathrm{g} / \mathrm{ml})$ for an additional $24 \mathrm{~h}$. Apoptosis was determined using the PI/Annexin-V as described in methods. The data represent the mean values $\pm \mathrm{SD}$ from 3 independent experiments. p-value: cells treated with a single agent (ch128.1Av or CDDP) vs. combined treatment with CDDP (Student's t-test).

The inhibition of the NF-кB pathway by ch128.1Av as shown above by Western blot analysis was also corroborated by using a DNA-binding assay for NF- $\mathrm{KB}$. Clearly, a concentration of $16 \mathrm{nM}$ was sufficient to significantly inhibit NF- $\mathrm{BB}$ DNA-binding activity in IM-9 and 8226 cells (Fig. 2B). The $\mathrm{NF}-\kappa \mathrm{B}$ inhibitor DHMEQ was used as an internal positive control. The role of NF- $\mathrm{KB}$-induced sensitization to CDDPinduced apoptosis was further confirmed by the use of the NF-кB inhibitor DHMEQ. Treatment of IM-9 (Fig. 2C, left panel) and 8226 (Fig. 2C, right panel) cells with different concentrations of DHMEQ (10 and $20 \mu \mathrm{M}$ ) resulted in significant potentiation of sensitization to CDDP-induced apoptosis. Single agents alone had no effect. These findings show that blocking NF- $\mathrm{KB}$ activity can lead to sensitization to CDDP-induced apoptosis, suggesting that NF- $\mathrm{KB}$-induced inhibition by ch128.1 Av is involved in the chemosensitization of malignant B-cells to CDDP-induced apoptosis.

Inhibition of the constitutively activated Akt pathway in malignant B-cells by ch128.1Av. In addition to inhibition of the NF- $\mathrm{KB}$ pathway by ch128.1Av shown above, the constitutively 
A

IM-9

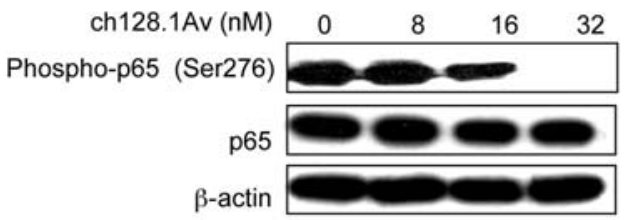

B

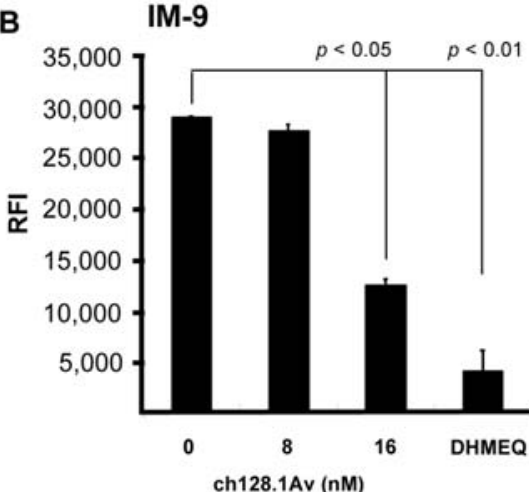

C

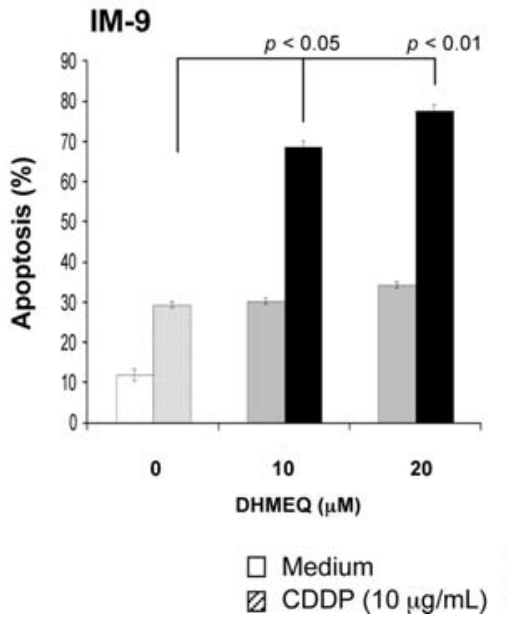

8226

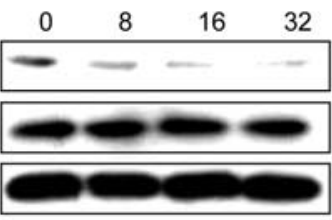

8226

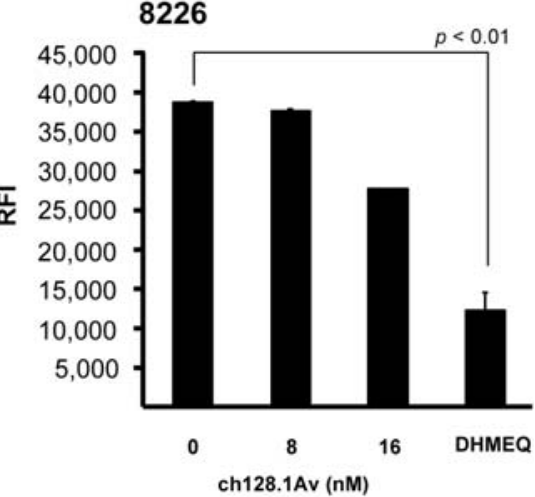

8226

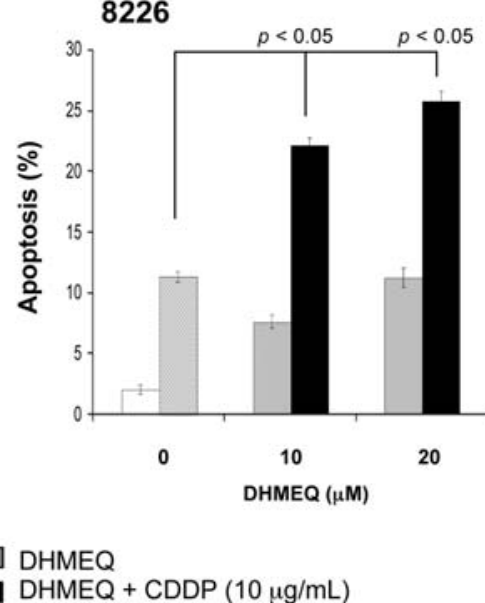

Figure 2. Inhibition of NF- $\mathrm{NB}$ by ch128.1Av and the role of NF- $\kappa \mathrm{B}$ in tumor cell sensitization to CDDP-induced apoptosis. (A) ch128.1Av inhibits NF- $\kappa \mathrm{B}$ activation as evidenced by the decreased level of phospho-p65 in IM-9 (left) and 8226 (right) cells. Cells were treated with 0-32 nM of ch128.1Av for $24 \mathrm{~h}$ and cell lysates were prepared and examined by Western blot analysis for phospho-p65, p65 and B-actin expression. Blots are representative of 3 independent but reproducible experiments. (B) NF- $\mathrm{BB}$ DNA-binding activity in IM-9 (left) and 8226 cells (right). Cells were treated with 0-32 nM of ch128.1Av for $24 \mathrm{~h}$ and cell lysates were prepared and examined for DNA-binding analysis using Trans AM DNA-binding analysis kit as described in Materials and methods. The NF-кB inhibitor, DHMEQ, was used as an internal positive control. p-value: medium control compared to treatment with $16 \mathrm{nM}$ of ch128.1Av (Student's t-test). (C) IM-9 (left) and 8226 cells (right) were treated with various concentrations of (-)-DHMEQ (0-20 $\mu \mathrm{g} / \mathrm{ml})$ for $2 \mathrm{~h}$ and treated with CDDP (10 $\mu \mathrm{g} / \mathrm{ml})$ for an additional $24 \mathrm{~h}$. Apoptosis was determined using the PI/Annexin-V method. p-value: single (-)-DHMEQ or CDDP treatment vs. combined treatment (Student's t-test).

activated Akt pathway is also involved in the regulation of resistance to apoptosis as well as its crosstalk with the NF-кB pathway (20). We, therefore, hypothesized that ch128.1Avinduced inhibition of $\mathrm{NF}-\kappa \mathrm{B}$ may have resulted from inhibition of the Akt pathway. Treatment of IM-9 and 8226 with various concentrations of ch128.1Av resulted in significant decrease of phospho-Akt (ser 473) levels with no effect on the total Akt levels (Fig. 3A). In IM-9 (Fig. 3A, left panel), treatment with $\geq 8 \mathrm{nM}$ ch128.1Av resulted in decreased phospho-Akt levels and there was complete inhibition at $32 \mathrm{nM}$. The concentration range of ch128.1 Av in the inhibition of phospho-Akt was in the same range as observed in the inhibition of NF- $\mathrm{B}$ seen in Fig. 2A. The effect of ch128.1Av on 8226 was less pronounced and the inhibition was observed at $32 \mathrm{nM}$ of
ch128.1Av (Fig. 3A, right panel). These results suggested that the inhibition of the Akt pathway by ch128.1Av may also be involved in the sensitization of malignant B-cell lines to CDDP apoptosis. To test this hypothesis, we used a specific inhibitor of the Akt pathway, namely, LY294002. Treatment of the cancer cells with various concentrations of LY294002 (10-50 $\mu \mathrm{M})$ and followed by treatment with CDDP (1 and $5 \mu \mathrm{g} / \mathrm{ml}$ ) resulted in significant enhancement of apoptosis. In IM-9 cells, treatment with $50 \mu \mathrm{M}$ LY294002 in combination with CDDP $(5 \mu \mathrm{g} / \mathrm{ml})$ resulted in significant potentiation of apoptosis (Fig. 3B, left panel). Likewise, in 8226 cells, treatment with $50 \mu \mathrm{M}$ of LY294002 resulted in significant enhancement of apoptosis in combination with CDDP (5 $\mu \mathrm{g} / \mathrm{ml})$ (Fig. 3B, right panel). 
A

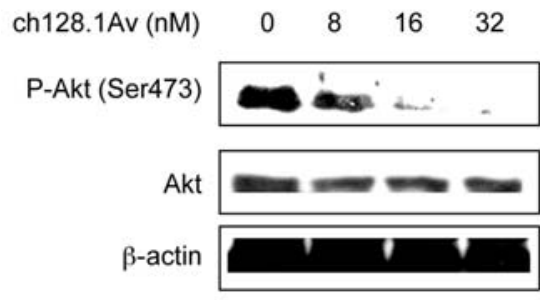

B

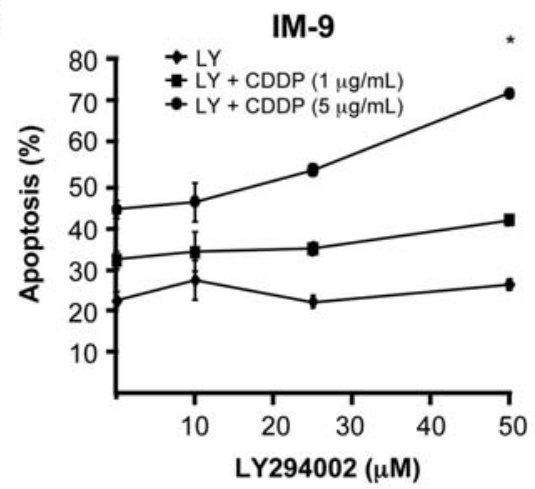

8226

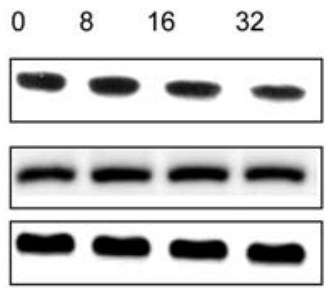

8226

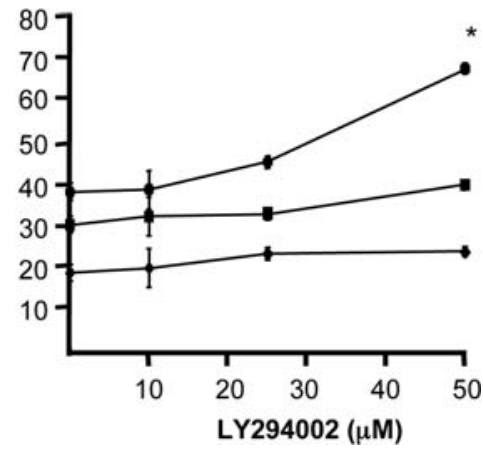

Figure 3. Inhibition of Akt activity by ch128.1Av and the direct role of Akt inhibition via LY294002 in the sensitization to CDDP-induced apoptosis. (A) The ch128.1 Av inhibits phospho-Akt in IM-9 cells (left) and 8226 cells (right). Cells were treated with 0-32 nM of ch128.1 Av for $24 \mathrm{~h}$ and cell lysates were prepared and examined by Western blot analysis for phospho-Akt, Akt and B-actin. Blots are representative of 3 independent but reproducible experiments. (B) Treatment of IM-9 cells (left) and 8226 cells (right) with a specific Akt inhibitor LY294002 results in sensitization to CDDP-induced apoptosis. IM-9 and 8226 cells were treated with various concentrations of CDDP $(1 \mathrm{or} 5 \mu \mathrm{g} / \mathrm{ml})$ for $24 \mathrm{~h}$ in the presence or absence of LY294002 (0-50 $\mu \mathrm{M})$ and apoptosis was determined using the PI/Annexin-V method. "p-value: single cell treatment with LY294002 or CDDP vs. combinational treatment. (Student's t-test).

A

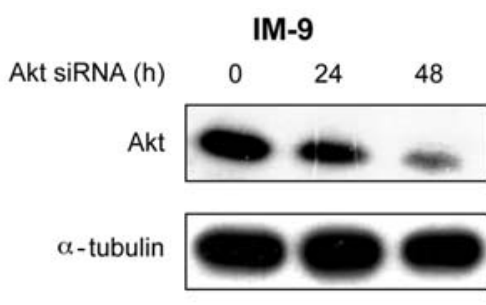

B

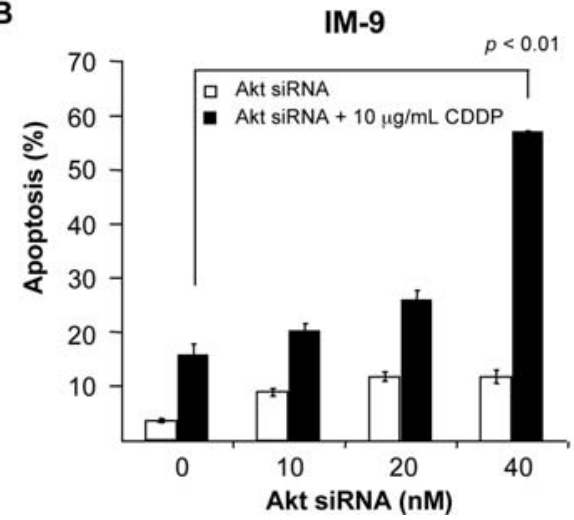

8226
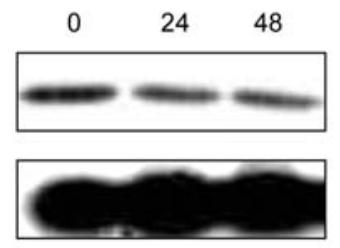

8226

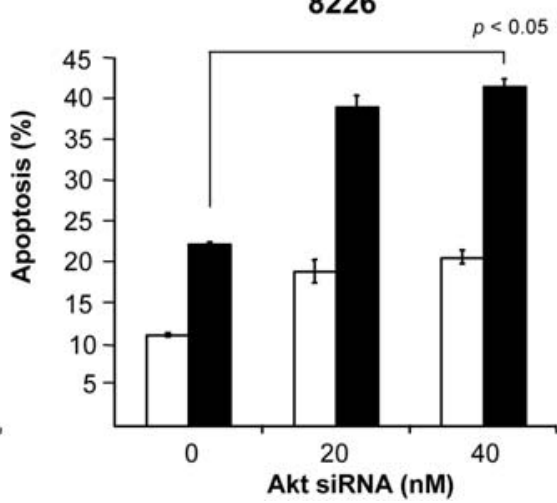

Figure 4. Direct inhibition of Akt by siRNA in cells results in sensitization to CDDP-induced apoptosis. (A) IM-9 (left) or 8226 (right) cells were treated with $8 \mathrm{nM}$ of either Akt siRNA or control scramble siRNA for 0-48 h. Cell lysates were examined for total Akt levels by Western blot analysis. $\alpha$-tubulin served as an internal control for loading. (B) Cells were treated with increasing concentrations of Akt siRNA for $48 \mathrm{~h}$ and CDDP (10 $\mu \mathrm{g} / \mathrm{ml})$ for an additional $24 \mathrm{~h}$, and apoptosis was determined using the PI/Annexin-V method. ${ }^{*}$-value: single Akt siRNA or CDDP treatment vs. combined treatment (Student's t-test).

The direct role of Akt in the sensitization to CDDP-induced apoptosis was confirmed by the use of Akt siRNA. IM-9 and 8226 cells were treated with Akt siRNA or control siRNA for
24 and 48 h. Shown in Fig. 4A (left panel) treatment of IM-9 cells with Akt siRNA resulted in significant inhibition of Akt as determined by Western blotting. Likewise, treatment of 
A

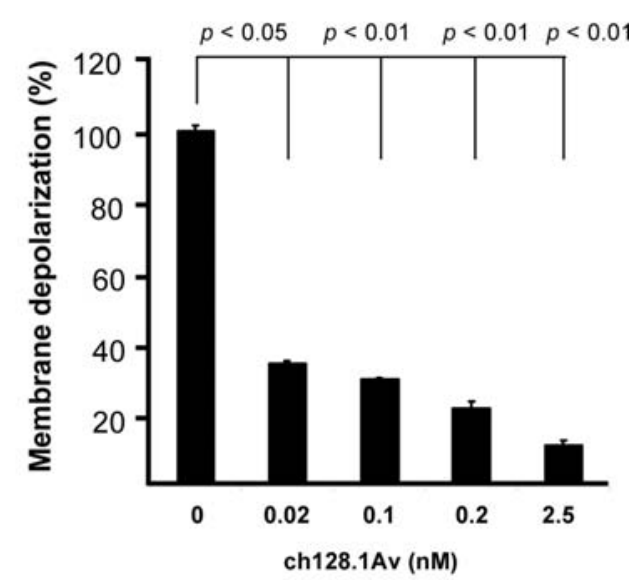

B

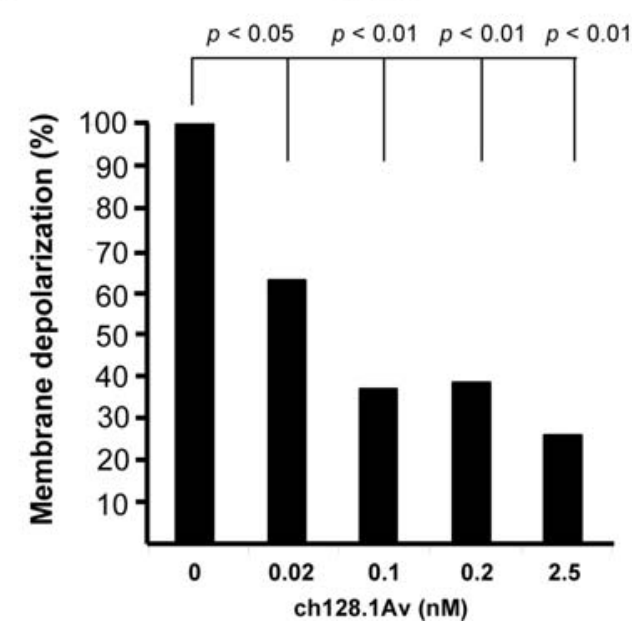

Figure 5. ch128.1Av-induces mitochondorial membrane potential depolarization in malignant B-cells. Mitochondorial membrane depolarization was assessed in IM-9 cells (A) and 8226 cells (B) using the DiOC $_{6}$ dye by flow cytometry as described in Materials and methods. The cells were treated with various concentrations of ch128.1 Av $(0.02-2.5 \mathrm{nM})$ for $24 \mathrm{~h}$ and then examined for membrane potential. Values are expressed as Mean Fluorescence Intensity (MFI) of $\mathrm{DiOC}_{6}$ incorporation. Untreated cells are represented as 100 and treated cells are calculated as percentage of untreated cells. The data represent the mean \pm SD of 3 independent experiments. p-value: medium control vs. treatment with $16 \mathrm{nM}$ of ch128.1Av (Student's t-test).

8226 with Akt siRNA also resulted in inhibition of Akt as shown in Fig. 4A, right panel. For sensitization to CDDP, IM-9 and 8226 cells were treated with Akt siRNA or control in the presence or absence of CDDP $(10 \mu \mathrm{g} / \mathrm{ml})$ and then examined for apoptosis. As seen in Fig. 4B treatment of IM-9 and 8226 with the combination of Akt siRNA and CDDP resulted in significant potentiation of apoptosis. These findings corroborate the findings observed above with the Akt inhibitor LY294002 that show that inhibition of the AKT pathway plays a role in CDDP-mediated cytotoxicity. These findings show that blockage of the Akt pathway can lead to chemosensitization and suggest that ch128.1Av-mediated inhibition of the Akt pathway, in addition to the NF- $\mathrm{BB}$ pathway, is also involved in the sensitization of malignant B-cells to CDDPinduced apoptosis.
A

IM-9

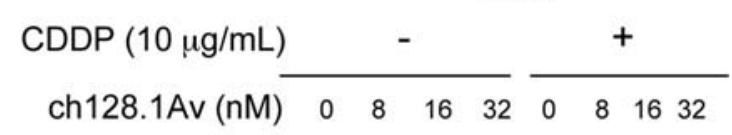

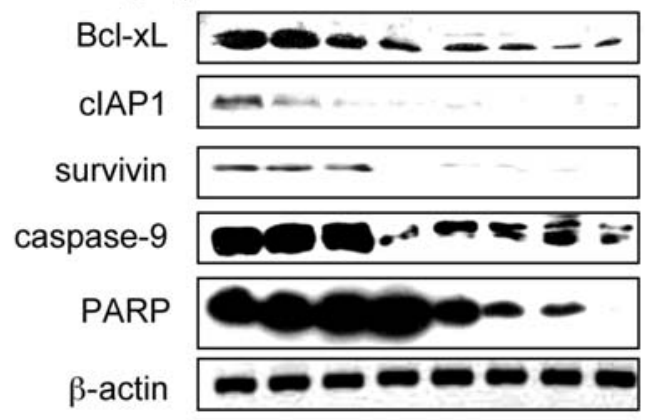

B

8226

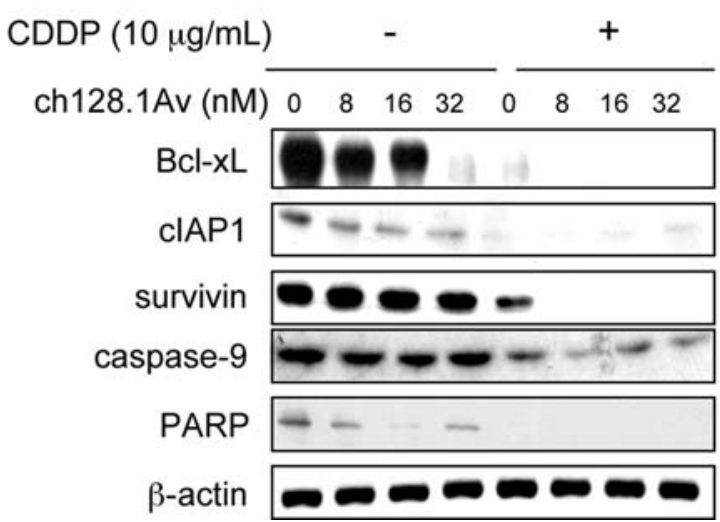

Figure 6. ch128.1Av sensitizes malignant B-cells to apoptosis by CDDP via activation of the intrinsic apoptotic pathway. Total cell lysates derived from IM-9 cells (A) and 8226 cells (B) treated with various concentrations of ch128.1Av (0-32 $\mathrm{nM})$ and CDDP $(10 \mathrm{mg} / \mathrm{ml})$ for $24 \mathrm{~h}$ were examined for levels of expression for BclxL, cIAP-1, survivin, caspase-9 and PARP by Western analysis. B-actin served as an internal control for loading. The blots are representative of three independent experiments.

Potential underlying mechanism of ch128.1Av-mediated sensitization of malignant $B$-cells to apoptosis by $C D D P$. We have previously shown that a high concentration of ch128.1Av $(50 \mathrm{nM})$ for treatment times of $48 \mathrm{~h}$ induces mitochondrial depolarization (13). Thus, we determined if the concentrations of ch128.1Av used in this study could also induce mitochondrial depolarization, which might play a role in its ability to sensitize cells to CDDP-mediated apoptosis. Cells were treated with various concentrations of ch128.1Av and examined for the mitochondrial membrane potential. Treatment with ch128.1Av resulted in significant mitochondrial membrane depolarization starting at concentrations of $0.02 \mathrm{nM}$ in both IM-9 (Fig. 5A) and 8226 (Fig. 5B) cells. These findings suggest that the membrane depolarization by ch128.1Av may result in the downstream activation of the apoptosome and in combination with CDDP results in the potentiation of apoptosis.

In order to examine the effects mediated by ch128.1Av downstream of the mitochondrion and the NF- $\mathrm{BB}$ and Akt pathways, whole-cell lysates from cells treated with various concentrations of ch128.1Av alone or in combination with 


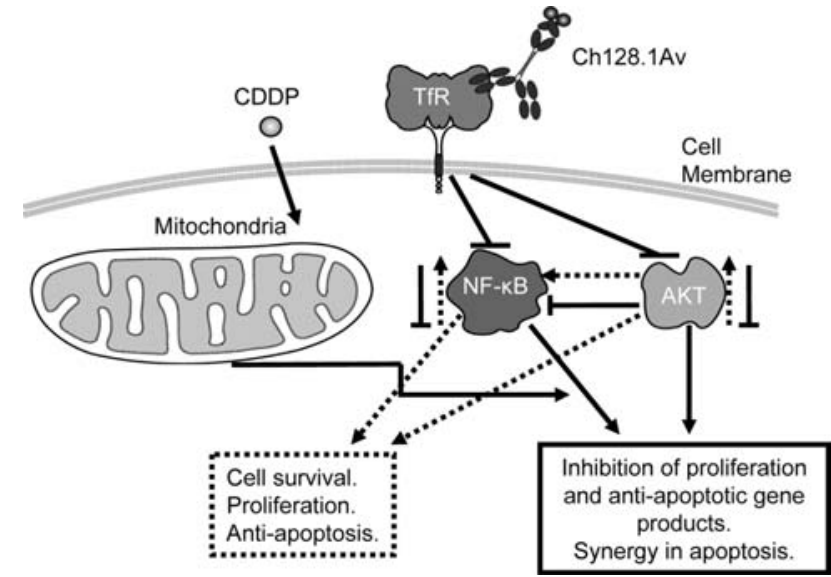

Figure 7. Schematic diagram of the mechanism by which ch128.1Av sensitizes malignant B-cells to apoptosis by chemotherapeutic drugs. Malignant B-cells constitutively express NF- $\mathrm{\kappa B}$ and Akt activating pathways These result downstream in the transcription and expression of various gene products that regulate cell survival, proliferation and resistance to apoptosis. The tumor cells overexpress the transferrin receptor. Upon binding of ch128.1Av to its corresponding transferrin receptor, the complex is internalized and several modifications take place in the cancer cells. Both the NF- $\mathrm{B}$ and Akt activating pathways are inhibited and consequently, inhibition of cell survival, proliferation and anti-apoptotic gene products are observed. In addition, ch128.1 Av also affects the mitochondria and depolarizes the membrane potential. Likewise, treatment with CDDP also affects the mitochondria, however, the combination treatment results in potentiation of apoptosis in the resistant malignant B-cells

CDDP were examined by immunoblotting for the expression levels of various proteins involved in these pathways. Treatment of IM-9 cells with ch128.1Av for $24 \mathrm{~h}$ resulted in the decrease of the anti-apoptotic gene products Bcl-xL, cIAP1 and survivin in the range of concentrations of 8-32 $\mathrm{nM}$ of ch128.1Av (Fig. 6A). There was also cleavage of caspase-9 but only at $32 \mathrm{nM}$ of ch128.1Av and there was no cleavage of PARP with ch128.1Av alone. The cleavage of caspase-9 observed here is consistent with previous findings showing caspase- 9 activity at concentrations above $10 \mathrm{nM}$ in IM-9 cells treated for more than $48 \mathrm{~h}$ (15). In IM-9 cells, the combination treatment of ch128.1Av with CDDP $(10 \mu \mathrm{g} / \mathrm{ml})$ resulted in significant decrease in the expression levels of Bcl-xL, cIAP1, and survivin (Fig. 6A). The combination treatment also showed caspase-9 and PARP cleavage (Fig. 6A). In 8226 cells, treatment with ch128.1v alone also decreased the expression levels of Bcl-xL, cIAP1 and PARP, but not survivin (Fig. 6B). The combination treatment totally inhibited the expression of all of these gene products (Fig. 6B). Cleavage of caspase- 9 and PARP were also increased by the combination treatment, consistent with apoptotic cell death. The downregulation of $\mathrm{Bcl}_{\mathrm{xL}}$ and survivin by ch1281-induced inhibition of the Akt pathway was corroborated by treatment with siRNA Akt (Fig. 4A). These findings support our contention that the sensitizing ability of ch128.1Av is mediated, in part, by the inhibition of anti-apoptotic gene products regulated by the $\mathrm{NF}-\kappa \mathrm{B}$ and Akt pathways.

\section{Discussion}

This study provides evidence, for the first time, that treatment of malignant B-cells with ch128.1Av inhibits the constitutive
$\mathrm{NF}-\kappa \mathrm{B}$ and Akt signaling pathways. This inhibition leads downstream to downregulation of anti-apoptotic gene products such as Bcl-xL and survivin and sensitized the cells to CDDP-induced apoptosis. The proposed model for the sensitization activity of ch128.1Av is shown in Fig. 7. The inhibition of NF- $\mathrm{KB}$ pathway was determined by decreased levels of phosphorylated p65. The inhibition of NF-кB activity and downregulation of anti-apoptotic gene products by ch128.1Av and chemosensitization was corroborated by the use of the NF- $\mathrm{B}$ inhibitor DHMEQ, which mimicked ch128.1 Av in the inhibition of NF- $\mathrm{KB}$ and chemosensitization to CDDP. Treatment of IM-9 and 8226 cells with ch128.1Av significantly inhibited the constitutive activity of the PI3K/Akt signaling pathway. Inhibition of this pathway resulted downstream in the inhibition of $\mathrm{Bcl}-\mathrm{xL}$ and survivin and sensitized the cells to CDDP-induced apoptosis. The role of Akt pathway in ch128.1Av-induced sensitization of cancer cells to CDDP-induced apoptosis was corroborated by the use of both the PI-3K inhibitor LY294002 and by the use of siRNA for Akt. Treatment of IM-9 and 8226 cells with LY294002 or Akt siRNA inhibited the Akt pathway and both $\mathrm{Bcl}-\mathrm{xL}$ and survivin expression and sensitized the cells to CDDP-induced apoptosis. The inhibition of Akt and NF-кB activities by ch128.1Av with the combination treatment with CDDP resulted in the activation of the mitochondrial apoptotic pathway and in the activation of caspase-9, PARP cleavage and apoptosis. These findings establish the potential therapeutic implication of ch128.1Av alone or in combination with subtoxic doses of chemotherapeutic drugs in drug-resistant malignant B-cells.

In mammals, the $\mathrm{NF}-\kappa \mathrm{B}$ contains five members: Rel-A (p65), Rel-B, c-Rel, NF-кB (p50 and its precursor p100) and NF-кB 2 (p52 and its precursor p100), the most abundant being the p65/p50 heterodimer (21). In normal cells, NF-кB

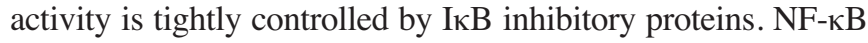
activation can be induced by a variety of stimuli resulting in the phosphorylation of IкB, ubiquitination and IкB degradation by the $26 \mathrm{~S}$ proteasome, allowing the translocation of NF-kB to the nucleus and activation of gene transcription. NF- $\mathrm{KB}$ phosphorylation is catalyzed by the multi-protein IKK complex, which phosphorylates and activates of the upstream NIK $(21,22)$. Here, we show a significant decrease of the phosphorylation state of p65 at less than $8 \mathrm{~h}$ following treatment, suggesting of a novel function of ch128.1 Av as a negative regulator of the $\mathrm{NF}-\kappa \mathrm{B}$ pathway.

$\mathrm{NF}-\kappa \mathrm{B} /$ Rel transcription factors bind to NF- $\mathrm{NB}$ control elements present in the promoter of a wide variety of target genes that regulate cellular differentiation, proliferation, survival and apoptosis $(21,22)$. Activation of the NF- $\mathrm{B}$ pathway is, in part, responsible for the transcriptional activation and expression of anti-apoptotic Bcl-2 family members which protect tumor cells from drug-induced apoptosis (21-23). In this study, we show that inhibition of the NF-кB pathway by ch128.1Av resulted in the down-regulation of Bcl-xL and survivin. We have reported that inhibition of $\mathrm{Bcl}-\mathrm{xL}$ is critical for chemosensitization $(23,24)$, and hence, our findings suggest that inhibition of Bcl-xL and survivin correlate with ch128.1Av-induced sensitization to CDDP.

Akt is involved in mediating various biological responses such as inhibition of apoptosis and stimulation of cell 
proliferation (20). Phosphorylation of Akt at serine or threonine residues activates the kinase activity of Akt. Activation of Akt can be mediated by various events, namely, binding of ligand to receptors on the cell membrane and causing autophosphorylation in the intracellular domain of the receptor. Thus, PI3k is recruited to the phosphothyrosine residues and becomes activated. In B-cells, PI3k is activated by BCR via SYK and BCAP (B-cell receptor associated protein). PI3k then phosphorylates membrane-bound PIP2 to generate PIP3. The binding of PIP3 to the PH domain anchors Akt to the plasma membrane and allows its phosphorylation and activation of PDK1. Also, Akt can be activated by cellular stress such as heat shock, UV, deschemia, hypoxia and oxidated stress (20).

The PI3K-Akt signaling pathway regulates many normal cellular processes and aberrant activation of the PI3K-Akt pathway has been widely implicated in many cancers (20). The PI3K-Akt pathway is a key regulator of cell survival through multiple downstream targets. IM-9 and 8226 cells exhibit constitutive activation of the Akt pathway. We have previously demonstrated that anti-CD20 monoclonal antibody, rituximab, inhibited the Akt pathway, leading to inhibition of Bcl-xL and ultimately to chemosensitization via the mechanism described above (23). Treatment of malignant B-cells with ch128.1Av resulted in decreased activation of the Akt pathway as well as depolarization of the mitochondrial membrane. However, little caspase- 9 activation was observed (as expected) with ch128.1Av treatment alone due to the short treatment time of $24 \mathrm{~h}$. Previous studies have shown activation of caspase- 9 and -3 in cells treated with ch128.1Av for more than $48 \mathrm{~h}(13,15)$. Our results demonstrate that treatment of IM-9 and 8226 cells with ch128.1Av in combination with CDDP significantly suppressed expressions of Bcl-xL, survivin and cIAP1. Taken together, the combined treatment of ch128.1Av with CDDP resulted in enhanced cytotoxicity that was stronger and occurred faster compared to ch128.1Av alone treated cells. In addition, the combination treatment resulted in the activation of the mitochondrial apoptotic pathway.

Various mechanisms contribute to the Akt pathway in tumors including perturbation of upstream tensen, homolog deleted in chromosome 10 (PTEN) and phosphatidylinositol triphosphate (PIP3). Others include autocrine or paracrine stimulation of receptor tyrosine kinases and overexpression of growth factor receptors and/or RAS activation. Targeting of Akt directly or indirectly inhibits cell proliferation, promotes apoptosis and/or reverses sensitivity to chemotherapy (20). Cross-talk between the PI3K and the NF- $\mathrm{KB}$ pathway has been reported in a number of systems (25). Thus, the observed findings of ch128.1Av inhibition of NF-kB and Akt may have resulted from this cross-talk. Although we can not rule out the involvement of other pathways, in the present study we show that both the NF-кB and Akt pathways play an important role in ch128.1Av-mediated chemosensitization of malignant B-cells to CDDP-induced cytotoxicity.

In conclusion, our findings here demonstrate, for the first time, that ch128.1Av is able to potentiate CDDP-induced apoptosis in IM-9 and 8226 cancer cells via inhibition of the constitutively activated Akt and NF-кB pathways and expression of downstream anti-apoptotic molecules. Thus, ch128.1Av-induced cytotoxicity is not only due to the iron deficiency, but also to the inhibition of anti-apoptotic molecules such as cIAPs, Bcl-xL and survivin. In addition, ch128.1Av induces mitochondrial membrane depolarization and the combination of ch128.1Av and CDDP through the activation of the intrinsic apoptotic pathway results in enhanced apoptosis. Our data suggest that ch128.1Av can effectively break the tumor cell resistance to CDDP at very low concentrations and could thereby support the clinical applicability of a combination of ch128.1Av and CDDP in the treatment of B-cell malignancies.

\section{Acknowledgements}

This study was supported by NIH/NCI grants R01CA107023, K01CA138559, R01 supplements CA107023-02S1 and CA57152-13S1, and the NIH/NCI training grant T32CA009120. The content is solely the responsibility of the authors and does not necessarily represent the official views of the National Cancer Institute or the National Institutes of Health.

\section{References}

1. Sloan B and Scheinfeld NS: Pazopanib, a VEGF receptor tyrosine kinase inhibitor for cancer therapy. Curr Opin Investig Drugs 9: 1324-1335, 2008.

2. Law CL, Gordon KA, Collier J, et al: Preclinical antilymphoma activity of a humanized anti-CD40 monoclonal antibody, SGN-40. Cancer Res 65: 8331-8338, 2005.

3. Lee JH, Koo TH, Yoon H, et al: Inhibition of NF-kappa B activation through targeting I kappa B kinase by celastrol, a quinone methide triterpenoid. Biochem Pharmacol 72: 1311-1321, 2006.

4. Watanabe M, Dewan MZ, Okamura T, et al: A novel NF-kappaB inhibitor DHMEQ selectively targets constitutive NF-kappaB activity and induces apoptosis of multiple myeloma cells in vitro and in vivo. Int J Cancer 114: 32-38, 2005.

5. Tatetsu H, Okuno Y, Nakamura M, et al: Dehydroxymethylepoxyquinomicin, a novel nuclear factor-kappaB inhibitor, induces apoptosis in multiple myeloma cells in an IkappaBalphaindependent manner. Mol Cancer Ther 4: 1114-1120, 2005.

6. Rizvi MA, Ghias K, Davies KM, et al: Enzastaurin (LY317615), a protein kinase Cbeta inhibitor, inhibits the AKT pathway and induces apoptosis in multiple myeloma cell lines. Mol Cancer Ther 5: 1783-1789, 2006.

7. Hayun M, Naor Y, Weil M, et al: The immunomodulator AS101 induces growth arrest and apoptosis in multiple myeloma: association with the Akt/survivin pathway. Biochem Pharmacol 72: $1423-1431,2006$

8. Schwartz RN and Vozniak M: Current and emerging treatments for multiple myeloma. J Manag Care Pharm 14: 12-19, 2008.

9. Daniels TR, Delgado T, Rodriguez JA, Helguera G and Penichet ML: The transferrin receptor part I: Biology and targeting with cytotoxic antibodies for the treatment of cancer. Clin Immunol 121: 144-158, 2006.

10. Habashy HO, Powe DG, Staka CM, et al: Transferrin receptor (CD71) is a marker of poor prognosis in breast cancer and can predict response to tamoxifen. Breast Cancer Res Treat (In press).

11. Daniels TR, Delgado T, Helguera G and Penichet ML: The transferrin receptor part II: targeted delivery of therapeutic agents into cancer cells. Clin Immunol 121: 159-176, 2006.

12. Ng PP, Dela Cruz JS, Sorour DN, et al: An anti-transferrin receptor-avidin fusion protein exhibits both strong proapoptotic activity and the ability to deliver various molecules into cancer cells. Proc Natl Acad Sci USA 99: 10706-10711, 2002.

13. Ng PP, Helguera G, Daniels TR, et al: Molecular events contributing to cell death in malignant human hematopoietic cells elicited by an IgG3-avidin fusion protein targeting the transferrin receptor. Blood 108: 2745-2754, 2006.

14. Rodriguez JA, Helguera G, Daniels TR, et al: Binding specificity and internalization properties of an antibody-avidin fusion protein targeting the human transferrin receptor. J Control Release 124: 35-42, 2007. 
15. Daniels TR, Ng PP, Delgado T, et al: Conjugation of an antitransferrin receptor IgG3-avidin fusion protein with biotinylated saporin results in significant enhancement of its cytotoxicity against malignant hematopoietic cells. Mol Cancer Ther 6: 2995-3008, 2007.

16. Ortiz-Sanchez E, Daniels TR, Helguera G, Martinez-Maza O, Bonavida B and Penichet ML: Enhanced cytotoxicity of an antitransferrin receptor IgG3-avidin fusion protein in combination with gambogic acid against human malignant hematopoietic cells: functional relevance of iron, the receptor, and reactive oxygen species. Leukemia 23: 59-70, 2009.

17. Helguera G and Penichet ML: Antibody-cytokine fusion protein for the therapy of cancer. Methods Mol Med 109: 347-374, 2005.

18. Span LF, Pennings AH, Vierwinden G, Boezeman JB Raymakers RA and de Witte T: The dynamic process of apoptosis analyzed by flow cytometry using Annexin-V/propidium iodide and a modified in situ end labeling technique. Cytometry 47: 24-31, 2002.

19. Huerta-Yepez S, Vega M, Jazirehi A, et al: Nitric oxide sensitizes prostate carcinoma cell lines to TRAIL-mediated apoptosis via inactivation of NF-kappa B and inhibition of Bcl-xl expression. Oncogene 23: 4993-5003, 2004.
20. Vivanco I and Sawyers CL: The phosphatidylinositol 3-kinase AKT pathway in human cancer. Nat Rev 2: 489-501, 2002.

21. Umezawa K: Inhibition of tumor growth by NF-kappaB inhibitors. Cancer Sci 97: 990-995, 2006.

22. Kikuchi E, Horiguchi Y, Nakashima J, et al: Suppression of hormone-refractory prostate cancer by a novel nuclear factor kappaB inhibitor in nude mice. Cancer Res 63: 107-110, 2003.

23. Suzuki E, Umezawa K and Bonavida B: Rituximab inhibits the constitutively activated PI3K-Akt pathway in B-NHL cell lines: involvement in chemosensitization to drug-induced apoptosis Oncogene 26: 6184-6193, 2007.

24. Jazirehi AR, Vega MI, Chatterjee D, Goodglick L and Bonavida B: Inhibition of the Raf-MEK1/2-ERK1/2 signaling pathway, Bcl-xL down-regulation, and chemosensitization of non-Hodgkin's lymphoma B cells by Rituximab. Cancer Res 64: 7117-7126, 2004.

25. Yin D, Woodruff M, Zhang Y, et al: Morphine promotes Jurkat cell apoptosis through pro-apoptotic FADD/P53 and anti-apoptotic PI3K/Akt/NF-kappaB pathways. J Neuroimmunol 174: 101-107, 2006. 